\title{
Remote sensing of cloud sides of deep convection: towards a three-dimensional retrieval of cloud particle size profiles
}

\author{
T. Zinner ${ }^{1,2}$, A. Marshak ${ }^{1}$, S. Lang ${ }^{3}$, J. V. Martins ${ }^{1,4}$, and B. Mayer ${ }^{2}$ \\ ${ }^{1}$ NASA - Goddard Space Flight Center, Climate and Radiation Branch, Greenbelt, MD, USA \\ ${ }^{2}$ Deutsches Zentrum für Luft- und Raumfahrt, Inst. für Physik der Atmosphäre, Oberpfaffenhofen, 82230 Wessling, Germany \\ ${ }^{3}$ NASA - Goddard Space Flight Center, Mesoscale Atmospheric Processes Branch, Greenbelt and Science Systems and \\ Applications Inc., Lanham, MD, USA \\ ${ }^{4}$ Department of Physics and Joint Center for Earth Systems Technology, University of Maryland Baltimore County, \\ Baltimore, MD, USA
}

Received: 10 December 2007 - Published in Atmos. Chem. Phys. Discuss.: 3 March 2008

Revised: 5 June 2008 - Accepted: 3 July 2008 - Published: 18 August 2008

\begin{abstract}
The cloud scanner sensor is a central part of a recently proposed satellite remote sensing concept - the three-dimensional (3-D) cloud and aerosol interaction mission (CLAIM-3D) combining measurements of aerosol characteristics in the vicinity of clouds and profiles of cloud microphysical characteristics. Such a set of collocated measurements will allow new insights in the complex field of cloudaerosol interactions affecting directly the development of clouds and precipitation, especially in convection. The cloud scanner measures radiance reflected or emitted by cloud sides at several wavelengths to derive a profile of cloud particle size and thermodynamic phase. For the retrieval of effective size a Bayesian approach was adopted and introduced in a preceding paper.

In this paper the potential of the approach, which has to account for the complex three-dimensional nature of cloud geometry and radiative transfer, is tested in realistic cloud observing situations. In a fully simulated environment realistic cloud resolving modelling provides complex 3-D structures of ice, water, and mixed phase clouds, from the early stage of convective development to mature deep convection. A three-dimensional Monte Carlo radiative transfer is used to realistically simulate the aspired observations.

A large number of cloud data sets and related simulated observations provide the database for an experimental Bayesian retrieval. An independent simulation of an additional cloud field serves as a synthetic test bed for the demon-
\end{abstract}

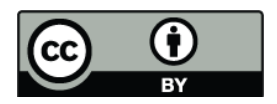

Correspondence to: T. Zinner (tobias.zinner@dlr.de) stration of the capabilities of the developed retrieval techniques. For this test case only a minimal overall bias in the order of $1 \%$ as well as pixel-based uncertainties in the order of $1 \mu \mathrm{m}$ for droplets and $8 \mu \mathrm{m}$ for ice particles were found for measurements at a high spatial resolution of $250 \mathrm{~m}$.

\section{Introduction}

Clouds play a critical role in the earth's energy balance and water cycle and are at the same time strongly influenced by the aerosol surrounding them. Since Twomey (1977) described the impact of aerosol on the concentration of cloud particles and the cloud albedo, an increasing number of possible indirect aerosol effects on cloud development and thus weather and climate have been found. For example, Albrecht (1989) describes possible implications of high aerosol load for a delay in precipitation onset and a prolonged stratocumulus life-time; or Kaufman and Koren (2006) present observations of a correlation between aerosol type and concentration and cloud cover.

In addition to changes in the radiation budget due to these interactions, the impact of aerosol properties on the convective cloud development is important as well. Convective development is highly sensitive towards the aerosol environment. At the same time convection is central for the global climate due to its role in the re-distribution of latent heat and the water-cycle. This is object of a series of investigations concentrated on the microphysical development of convective clouds and its dependence on the aerosol environment (e.g. Williams et al., 2002; Rosenfeld and Woodley, 2000;

Published by Copernicus Publications on behalf of the European Geosciences Union. 


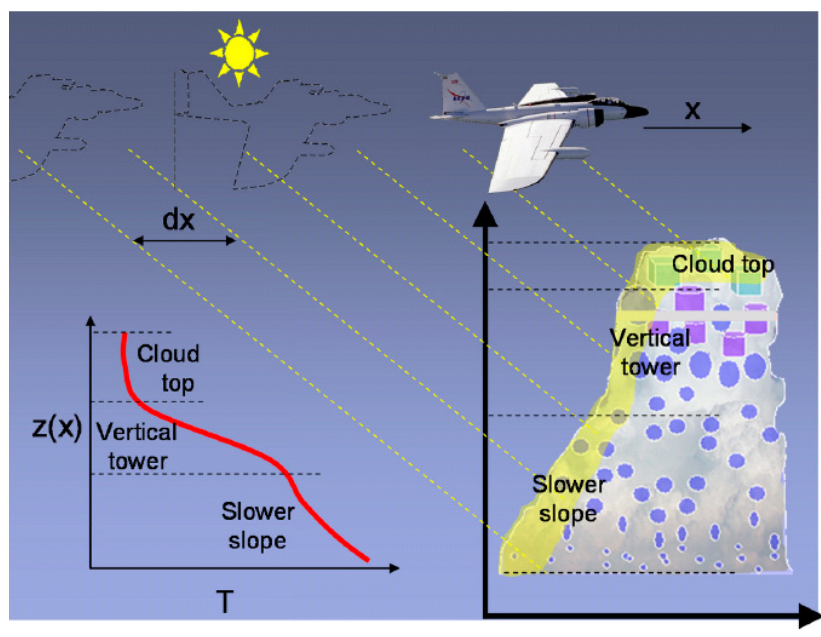

Fig. 1. Schematic depiction of the cloud scanner concept (taken from Martins et al., 2007): the sun is behind the sensor in the plane of observation. Reflected solar and near infra-red radiance is used to measure the particle size and phase of the outer layer of a convective cell. The measurement of thermal radiance provides height and additional geometrical information.

Andreae et al., 2004; Rosenfeld, 2006). Therefore, the recent IPCC 2007 again marks aerosol-cloud interaction as one of the fields with highest uncertainty regarding climate change.

At the same time, today's cloud and aerosol microphysical measurements, and with them our understanding, still have severe limitations, especially as far as convection is concerned. In-situ data, already limited in spatial coverage, is difficult to measure in highly turbulent cloud environment and for deep convection its collection is always related to high risk. Satellite based passive remote sensing naturally concentrates on the cloud top while ground based passive remote sensing provides only limited information as well, especially for optically thick clouds. Active remote sensing relies on strong assumptions and is limited in spatial accuracy. For these reasons Martins et al. (2007) proposed the threedimensional cloud and aerosol interaction mission (CLAIM3D) to contribute new insights through collocated aerosol and cloud microphysical measurements.

A central part of CLAIM-3D is an innovative approach, the so-called cloud scanner instrument (see Fig. 1), for the observation of profiles of cloud phase and particle size which reflect the impact of aerosol on the development of convection and precipitation. It replaces the classical satellite observational approach of cloud top remote sensing by cloud side remote sensing. Solar radiation reflected from cloud sides and their thermal emission is observed in several spectral regions from a slanted viewing geometry by either satellite, aircraft or ground based sensors. The collection of information along the vertical axis is either realized by a scanning mechanism or through the motion of an air- or spacecraft us- ing a fixed viewing zenith angle. This way cloud profile information inaccessible under the classical approach becomes available to passive remote sensing. From a combination of a visible (VIS), two near infra-red (NIR), and a thermal infrared (TIR) channel, profiles of cloud phase and cloud particle size can be derived.

To an even larger extent than the classical passive remote sensing, the cloud side observations are susceptible to threedimensional (3-D) effects like the strong dependence of reflectivity on the varying illumination of the complex small scale structure of cloud sides. These issues were discussed in detail in Marshak et al. (2006). A central problem are shadows. Without detailed knowledge of the cloud structure, these introduce unpredictable variation of reflectance. For our approach, this issue is minimised through a limitation to observations of the backscattered solar radiation, i.e. the sensor view is selected in a way that the sun is laterally "in the back" of the sensor and vertically within $15^{\circ}$.

Cloud phase can be determined from a combination of reflectance in two NIR channels (centre wavelengths at 2.1 and $2.25 \mu \mathrm{m}$; Martins et al., 2007). A change in the imaginary part of the refractive index of ice between 2.1 and $2.25 \mu \mathrm{m}$, while the one for liquid water remains largely constant, allows for a clear identification of cloud phase from the ratio of reflectance in both channels. The retrieval of cloud particle size is based on the well known concept of Nakajima and King (1990). The sensitivity of the absorption at a NIR wavelength to particle size in combination with a VIS wavelength insensitive to particle size is widely used to derive effective particle size and optical thickness (e.g. Platnick et al., 2003). Due to the strongly slanted viewing geometry in the case of the cloud side observation optical thickness cannot be easily interpreted and only the cloud effective particle size is the only quantity retrieved in the following. Using the thermal information from the TIR channel the observations of cloud phase and effective size can be assigned to a certain height in the atmosphere given a suited atmospheric sounding in the vicinity of the observation.

The information on phase and effective size, retrievable from solar reflectance, is a function of the cloud characteristics close to the cloud edges depending on the 3-D photon transport, e.g. the penetration depth of photons into the cloud volume. Of decisive importance for the cloud scanner approach are therefore two questions: (1) How representative is information gained from the thin outer shell of the cloud for the inner part of the cloud (compare Fig. 1)? A typical penetration depth of photons at NIR wavelength is an optical thickness around 2 to 5, i.e. a few hundred meters (Marshak and Davis, 2005; Zinner and Mayer, 2006). (2) Is there a solution for the inversion of radiative transfer systematically linking observed values of reflectance to cloud properties for this situation of highly complex cloud structures and 3-D radiative effects?

Only if the effective radius doesn't change substantially with distance from the cloud edge, measurements of 
conditions at the edge can be representative for the main body of the cloud cell and a meaningful effective radius profile could be retrieved. The first question is thus strongly related to the mixing of cloudy and clear air at the boundary (the entrainment). Does it happen in a "homogeneous" or "inhomogeneous" way? This question not be answered conclusively in this paper, because as mentioned above reliable data is scarce, but a number of publications provide evidence that in a large number of situations effective radius is sufficiently constant with distance from the cloud edge (Blyth and Latham, 1991; Rosenfeld and Woodley, 1998; French et al., 2000; Freud et al., 2008; Gerber, 2006). This topic is object of Sect. 2.2.1.

Core of this paper will be the second question regarding the cloud scanner retrieval concept: is a statistical retrieval of cloud properties feasible at all for the complex interrelation of high resolution cloud structure and 3-D radiation field for the wide range of cloud structures existing in nature? Marshak et al. (2006) initially addressed it by testing their approach using statistically generated cloud structures containing simplified cloud microphysical properties. Here a more systematic proof-of-concept will be conducted based on a large number of convective cloud cases from cloud resolving modelling (CRM) with realistic detail in cloud structure and microphysical properties covering a wide range of natural possibilities. The significance of a radiative signature will be investigated by sampling of different cloud realisations. A database large enough to obtain dependable statistical results is formed and the related theoretical reliability of the retrieval is estimated quantitatively for realistic cloud situations.

The topics are addressed using two main tools: the Goddard Cumulus Ensemble model, a state-of-the-art CRM providing 3-D fields of cloud properties (Sect. 2.1, Tao et al., 2003), and the 3-D Monte Carlo radiative transfer code MYSTIC (Sect. 3, Mayer, 1999, 2000) for the simulation of accurate radiation fields related to these CRM cloud fields. In Sect. 2.2 parameterisations are introduced to complement the CRM output with values of particle effective size not provided by the model. Section 4 gives some examples of the simulations (for four wavelengths in the VIS, NIR and TIR spectral range) and a broader summary for the generated dataset of synthetic observations. A description of this database as basis of a retrieval is given in Sect. 5. A synthetic test case to demonstrate the capabilities of the approach is presented in Sect. 6. The conclusions describe the status and future of the cloud scanner concept. An Appendix presents the introduction of the delta-scaling approach into our Monte Carlo radiative transfer code as a necessary optimisation to meet the high computational demand of building a retrieval database of 3-D simulated radiance for a large number of cloud scenes.

\section{Cloud resolving simulation of deep convection}

Cloud structures from cloud resolving modelling (CRM) are often used as the basis for radiative transfer simulations (e.g. Barker et al., 2003; Cahalan et al., 2005). Their advantage over the use of statistical model data (e.g. Marshak et al., 2006) is the physical consistency of the output fields regarding structure, cloud dynamics, and cloud microphysics. Most models use so-called bulk microphysics parameterisations where the cloud particle volume is characterised in terms of mass content of different cloud particle classes (liquid or ice, cloud or precipitation). Others use elaborate spectral microphysics models explicitly simulating the development of cloud particle size distributions in these classes (e.g. Khain et al., 2001). Although the latter model group has the advantage of greater physical detail especially with respect to cloud optical properties, they are so far only used for simulations of limited spatial extent due to their large computational demand: e.g. small scale cloud scenes of boundary layer cloud types, or 2-D cross sections for extensive cloud systems like deep convection. As 3-D spatial variability of cloud structure and extensive 2-D radiance observations of deep convection are the object of our work, a bulk microphysics model is used. A description of the CRM and its bulk microphysics are given in the next section.

\subsection{Goddard Cumulus Ensemble model}

A realistic cloud basis for all of the subsequent simulated observations is provided by the 3-D version of the Goddard Cumulus Ensemble model (GCE). The model is described in Tao and Simpson (1993) and Tao et al. (2003). The model can produce 3-D cloud fields on domains large enough to facilitate the development of full-scale deep convective systems. At the same time it provides the spatial variability needed to develop the cloud scanner retrievals in a realistically complex environment. Output quantities include 3-D fields of temperature and pressure as well as the mass contents of the different cloud species: cloud ice, cloud liquid water, rain, snow, and graupel. Results are obtained for the 23 February 1999, a case that fell within the Tropical Rainfall Measuring Mission Large-Scale Biosphere-Atmosphere (TRMM LBA) experiment in Amazonia. This case is characterised by diurnally growing weak to moderate convection that then loosely organised into transient lines parallel to the deep tropospheric wind shear. The model domain consisted of $256 \times 256$ horizontal points at 250 -m resolution (equivalent to $64 \mathrm{~km} \times 64 \mathrm{~km}$ ) and 41 stretched vertical layers with height increments ranging from $37 \mathrm{~m}$ near the surface to $1028 \mathrm{~m}$ at the top and a total depth of about $23 \mathrm{~km}$. See Lang et al. (2007) for a description of the GCE model applied to this case.

Simulations were made using two different improved versions of the graupel-based microphysical scheme, which is largely based on Rutledge and Hobbs (1984). In addition to 

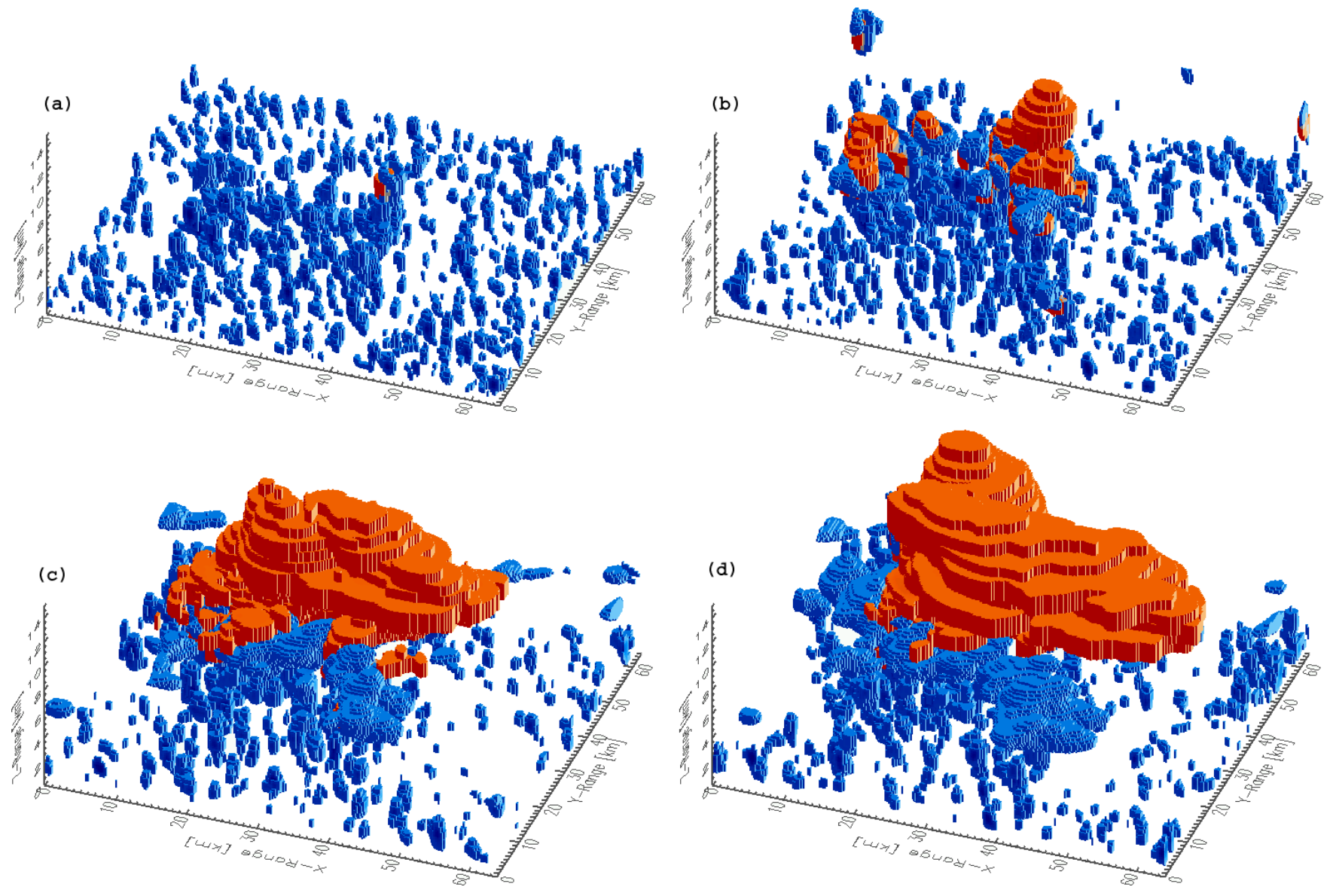

Fig. 2. GCE 3-D cloud scenes, domain size is $64 \mathrm{~km} \times 64 \mathrm{~km} \times 22.4 \mathrm{~km}$, horizontal resolution $250 \mathrm{~m}$, 41 vertical levels of variable thickness. Used output fields are cloud liquid water content (blue), cloud ice water content (red). Four examples are shown for 200, 240, 280, and 300 min after simulation start.

the improvements reported in Lang et al. (2007), in the first run the microphysics were further enhanced by: (1) scaling the Bergeron process for snow growth by the super saturation with resect to ice and water, (2) adding a simple rime splintering scheme (e.g. Hallett and Mossop, 1974), replacing the Fletcher (1962) curve for the concentration of ice crystals with the Meyers et al. (1992) formulation where appropriate, allowing for ice super saturation and the sublimation of precipitation-sized ice particles, and adding a sedimentation term for cloud ice based on Hong et al. (2004). In the second run, in addition to the previous modifications, the snow intercept is allowed to vary as a function of temperature similar to Hong et al. (2004) and cloud water is assumed to be frozen at temperatures below $-18^{\circ} \mathrm{C}$, as observed by Stith et al. (2002).

Cloud field output from the GCE simulation with the first microphysical setup for 13 points in time are provided (between 180 and 300 min after initialisation) as well as a single cloud field generated using the second microphysical setup (230 min after initialisation). As input for the radiative transfer simulations only the fields of cloud ice water content (IWC) and cloud liquid water content (LWC) are used (Fig. 2) and for this purpose translated to a regular 3-D grid of $250 \mathrm{~m} \times 250 \mathrm{~m} \times 200 \mathrm{~m}(\Delta x \times \Delta y \times \Delta z)$. Precipitation-sized particles (i.e. snow, rain, and graupel) are not included as these properties have minimal radiative impact for the spectral range of the observations simulated within this study.

\subsection{Parameterisations of cloud particle size}

As described above, the GCE output does not yet represent a complete input data set for the radiative transfer simulations, because it only provides mass content and phase of cloud particles but no size information. For a complete description of radiative effects, detailed information on cloud particle characteristics (cloud droplets, ice habit) and particle size distribution (or effective radius) is required to determine the full scattering and absorbing characteristics. The missing particle size (a main object of the cloud scanner retrieval) has to be established by other means. Using parameterisations, a 
realistic range of particle sizes is generated in a way physically consistent with the given cloud mass contents.

\subsubsection{Cloud droplets}

For the liquid water cloud part the assumption of a semiadiabatic cloud droplet effective radius is adopted. Cloud droplets are assumed to grow through condensation in a predominantly adiabatic way as they are lifted above the cloud base. The actual liquid water content can clearly differ from the theoretical adiabatic liquid water content due to entrainment, especially for turbulent convective cloud development. The particle size can be affected by entrainment as well.

"Homogenous mixing" and "inhomogeneous mixing" describe the two theoretical extreme possibilities (e.g. Baker et al., 1980): As cloud free air is mixed with cloudy air all droplets in a typical droplet size distribution evaporate according to the reduced humidity (homogenous mixing) and the average cloud particle size is decreasing. Heterogenous mixing takes place without changes of the droplet size distribution as cloud free air and cloudy air is not mixed on a microphysical scale. Cloud humidity conditions are sustained in "pockets" of droplet air while clear conditions prevail in "pockets" of droplet free air at least over a certain period of time.

Using laboratory experiments Baker et al. (1980) argue that the time constant of turbulent mixing is much larger than that of droplet evaporation, leading to independent areas of different regimes in the cloud volume. Blyth and Latham (1991) and French et al. (2000) found in in-situ measurements that droplet effective radius in cumulus clouds essentially depends on the height above cloud base and is not affected by entrainment. Moreover, Blyth and Latham (1991) show that the effective radius is clearly related to the theoretical adiabatic value. Gerber (2006) also finds an effective droplet size constant for a given height in the cloud and emphasises that such an insensitivity to entrainment could also be explained by completely homogenous mixing of near saturated air which would not affect the effective droplet size either. In-situ data is rarely collected once convection reaches a more vigorous stage. Freud et al. (2008), nonetheless, corroborate the assumption of effective radius being a pure function of height throughout the liquid part of growing deep convection. Rosenfeld and Woodley (1998) provide further support by means of in-situ and remote sensing and state that effective radius seems to be a function of height at least as long as no precipitation has developed.

Although neither the purely heterogenous mixing, nor the opposite purely homogeneous mixing can be expected in nature (see e.g. Gerber, 2006), a strong dependence of droplet effective size on the height above the cloud base is often observed. This leads to the following parameterisation of an adiabatic droplet effective radius. An adiabatic condensation rate depending on height and a respective theoretical adiabatic liquid water content $w_{\text {ad }}$ for each height can be derived from values of temperature and pressure at cloud bottom height (see e.g. Brenguier et al., 2000; Zinner et al., 2006). Using $w_{\text {ad }}$, a value for an adiabatic effective radius can be calculated using a fixed number concentration of cloud condensation nuclei $\mathrm{N}$

$w_{\mathrm{ad}}=\rho_{\mathrm{w}} N \frac{4}{3} \pi r_{\mathrm{vol} \mathrm{ad}}^{3}$

where $r_{\mathrm{volad}}$ is the theoretical volume average adiabatic droplet size, $\rho_{\mathrm{w}}$ is the density of liquid water. $r_{\mathrm{vol} \text { ad }}$ can be related to an actual sub-adiabatic effective radius

$r_{\text {eff }}=s r_{\text {eff ad }}=\frac{s}{k^{\frac{1}{3}}} r_{\text {vol ad }}$

where the factor $k$ is the typical ratio $r_{\mathrm{vol}}^{3} / r_{\mathrm{eff}}^{3} . s$ describes the ratio of an actually sub-adiabatic effective radius to the perfect adiabatic value $\left(s=r_{\text {eff }} / r_{\text {eff ad }}\right)$. This way, the influence of homogeneous mixing not considered by the pure adiabatic approach is represented by $s$. $k$ depends on the exact shape of the droplet spectrum. Here a typical value of $k=0.8$ derived from observed droplet spectra is used (Martin et al., 1994). For the adiabaticity $s$ of the effective radius of droplets, Blyth and Latham (1991) find values between 0.7 and 0.97. For the two realizations of CRM microphysics used in the following (see Sect. 2.1), two different values of $s_{1}=0.9$ (for the main database) and $s_{2}=0.8$ (for the test case in Sect. 6) are chosen to create two sets of cloud microphysics.

This way a semi-adiabatic value of effective radius is calculated for each grid box in the GCE field containing liquid water. It has to be emphasized that the theoretical adiabatic water content is only used as a way to derive this droplet size. The effective radius is used together with the original CRM simulated LWC values to form the cloud microphysical datasets. In turn, these two parameters define a value for a droplet number for each box. This number is not to be confused with the constant $\mathrm{N}$ used above which was related to the theoretical adiabatic LWC. Its values vary between several 10 (for regions of low CRM simulated LWC) and about 1000 drops per cubic centimetre (for large LWC values).

To relax our postulation of a constant effective radius for a given height and thus account for natural variability, statistical deviations from the mean are included. Gaussian noise with a standard deviation of the size of the vertical difference between two layers is added to each horizontal layer of effective radius values. Figure $3 b$ shows an example for the derived droplet effective radius for a vertical cross section through one of the CRM fields.

\subsubsection{Ice particles}

The effective radius of ice particles is known to depend on ice water content and temperature (e.g. Wyser, 1998; McFarquhar et al., 2003). In in-situ measurements ice particles are found to be larger the greater the IWC and the warmer the ambient temperature is. The latter is often attributed to either 


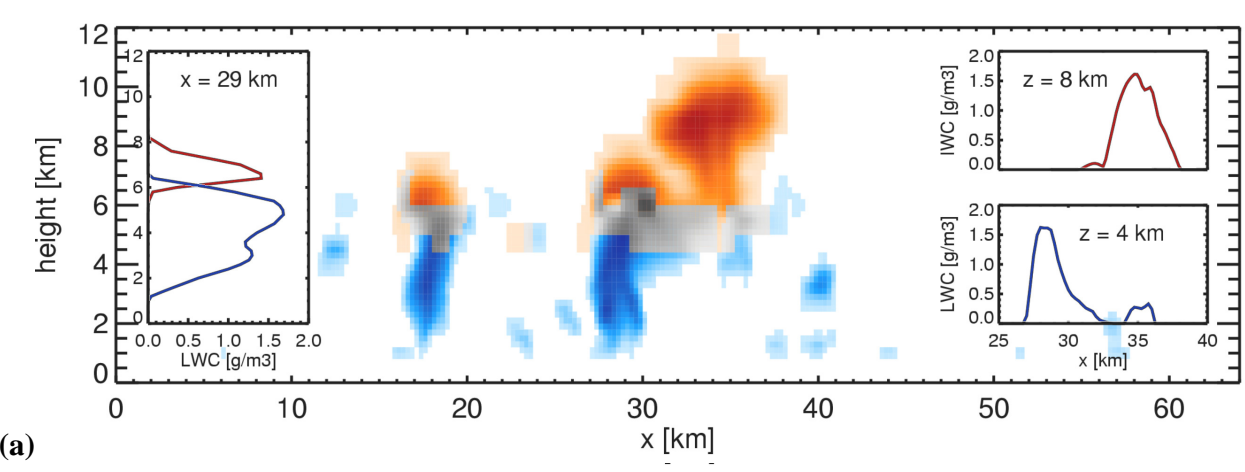

LWC $\left[\mathrm{g} / \mathrm{m}^{3}\right]$ IWC $\left[\mathrm{g} / \mathrm{m}^{3}\right]$

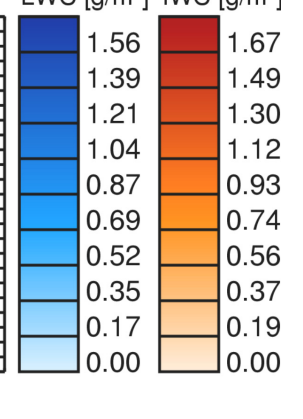

(a)

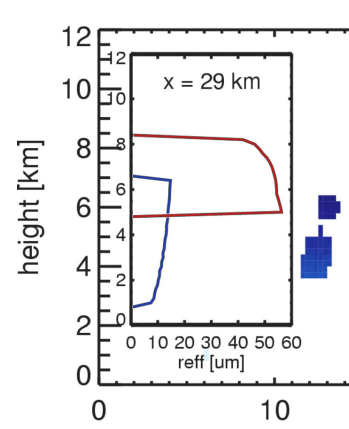

(b)
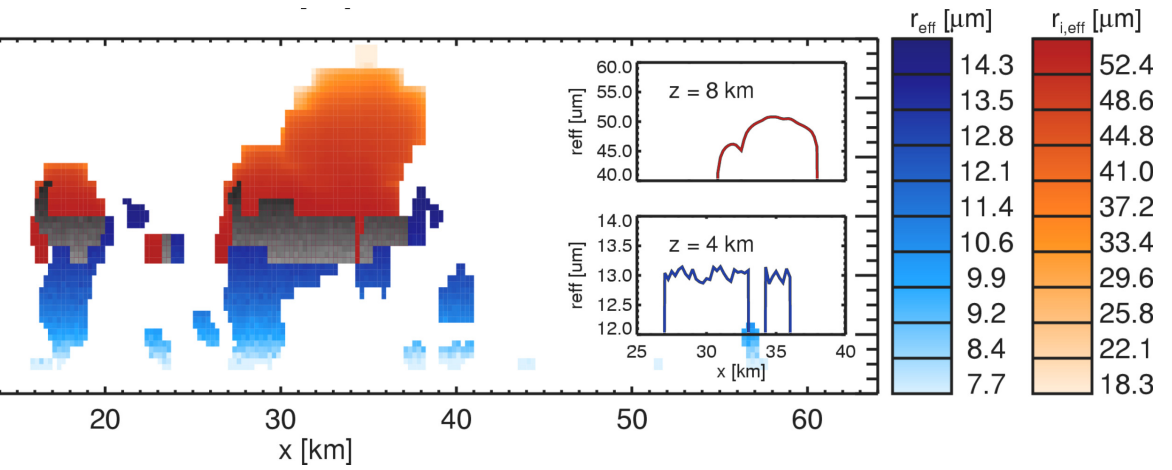

Fig. 3. (a) cross section through the GCE cloud mass content shown in Fig. $2 \mathrm{~b}$ at $y=32 \mathrm{~km}$, LWC in blue, IWC in red, mixed in grey, (b) cross section showing the effective particle size for the mass content in (a) after the parameterisations discussed in the text. For ice (red) and liquid water (blue) the small inserted graphs show vertical cross sections of the data at $x=29 \mathrm{~km}$ (left) and horizontal cross sections at $z=4$ and $8 \mathrm{~km}$ (for $25 \mathrm{~km}<x<40 \mathrm{~km}$, right).

sedimentation of larger particles in aging cirrus or to the successive activation of new ice nuclei (and thus the production of small particles) during convective ascent of an air parcel into colder and colder regions of the atmosphere.

For climate models several parameterisations of ice particle size can be found (see e.g. the compilation in McFarquhar et al., 2003). We chose the one after Wyser (1998) as it is on the one hand accounting for both the dependencies on temperature and ice water content, and on the other hand produces a large variety of ice effective radius values. It consists of a number of empirical equations representing dependencies found in in-situ data. Figure $3 b$ shows the result for a given cross section of IWC and temperature from GCE. Analogous to the adiabaticity factor for the droplet effective radius, a factor $s_{\text {ice }}$ is used to vary the dependence of ice particle size on IWC and temperature linearly for the two GCE data sets $\left(s_{\text {ice } 1}=1, s_{\text {ice } 2}=1.2\right)$.

Using the parameterisations described in this section, a 3D data set effective particle radius is obtained for the fields of cloud water and cloud ice content at each time step of the GCE model (Fig. 3). The different classes of precipitation from the GCE are neglected as their mass content is generally much smaller and precipitation particle sizes are much larger. Thus their contribution to the overall optical thickness is minimal and their impact on the radiative transfer in the visible and near infra-red spectral range is negligible.

\section{3-D radiative transfer model MYSTIC}

Our 3-D radiative transfer model is MYSTIC - a state of the art 3-D Monte Carlo (Mayer, 1999, 2000) continously tested and extended during the Intercomparison of 3-D Radiative Transfer Codes (I3RC, Cahalan et al., 2005). It is part of the radiative transfer package libRadtran (Library of Radiative Transfer, Mayer and Kylling, 2005).

The existing version of MYSTIC was extended and optimised as follows. For the given need to simulate a large amount of synthetic observations for several wavelength regions, the Monte Carlo technique reaches its computational limits even with today's computing capacities. A recently developed backward Monte Carlo mode (Emde and Mayer, 2007) was extended to cover the thermal wavelength region. The treatment of solar radiative transfer was considerably accelerated implementing the delta-scaling approximation into the code (Antyufeev, 1996; Iwabuchi, 2006). For details and tests see the Appendix.

\section{Synthetic observations}

13 cloud scenes of convection through various stages of their life cycle, from small pure liquid water cumuli to the mature anvil capped stage, form the cloud basis of the simulations. 

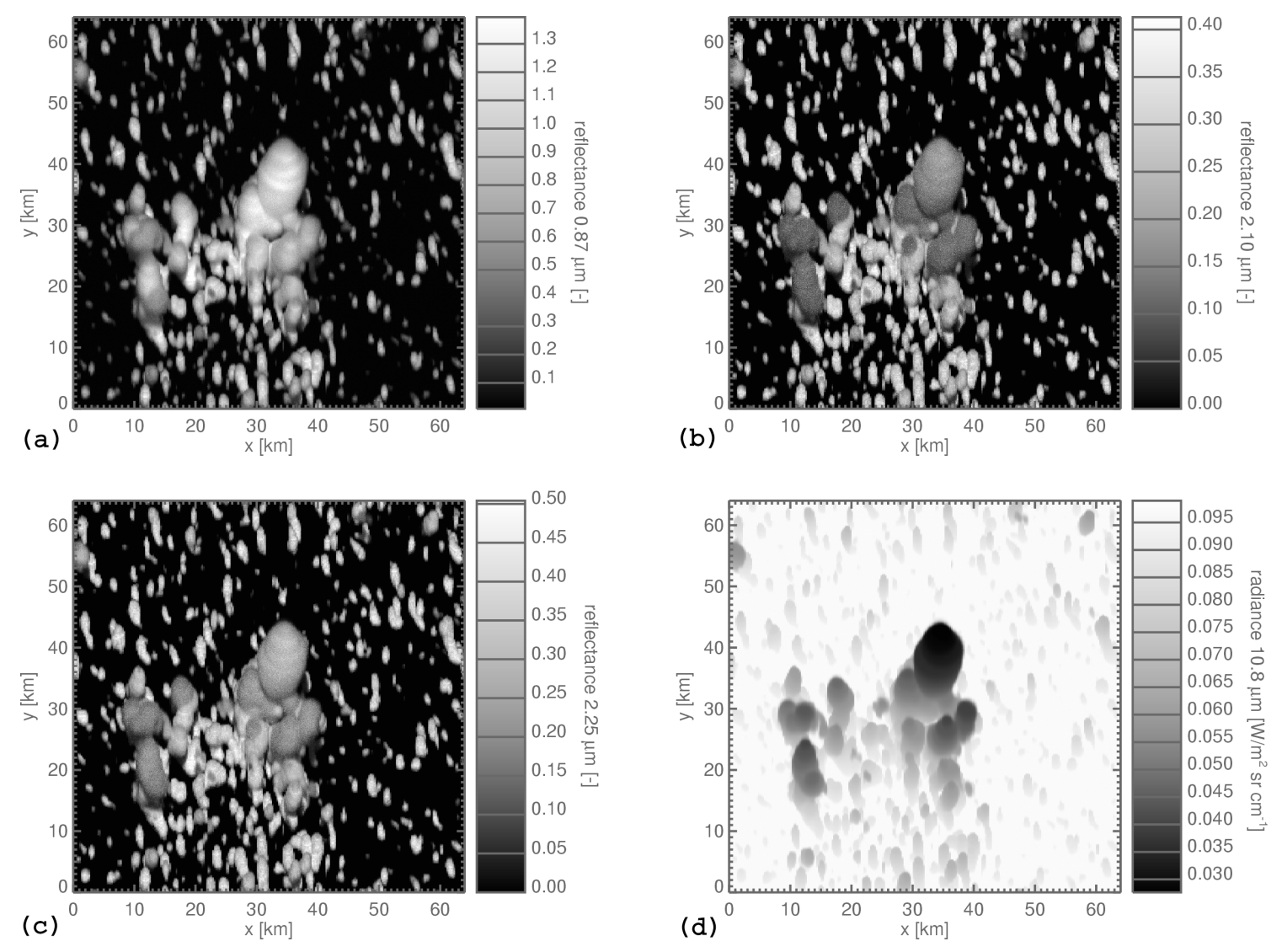

Fig. 4. Simulated cloud scanner observations: Based on GCE cloud data (compare Fig. 2) monochromatic reflectance for 0.87 (a), 2.1 (b), and $2.25 \mu \mathrm{m}$ (c), as well as emitted monochromatic radiance for $10.8 \mu \mathrm{m}$ (d). Solar zenith angle $45^{\circ}$, viewing zenith angle $60^{\circ}$. The lack of shadows is due to the fact that both sun and sensor have an azimuth of $180^{\circ}$ ("south").

One combination of solar zenith angle and sensor zenith angle is selected: $\theta_{\text {solar }}=45^{\circ}$ and $\theta_{\text {sensor }}=60^{\circ}$. The relative azimuth between both sun and sensor is 0 . That means, lateral shadows are impossible while vertical shadows can appear as the observing perspective is shallower than the solar illumination. The number of independent cloud data sets is quadrupled rotating each of the cloud data sets by $90^{\circ}, 180^{\circ}$, and $270^{\circ}$ around the vertical axis giving a total number of 52 independent convective cloud cases.

Representative for the four sensor channels, four single wavelength simulations are conducted: 0.87, 2.1, 2.25, and $10.8 \mu \mathrm{m}$. $0.87 \mu \mathrm{m}$ is dominated by scattering of solar radiation, and hence simply called the "visible" wavelength, while 2.1 and $2.25 \mu \mathrm{m}$ are sensitive to absorption by water and ice particles, the near infra-red wavelengths. The thermal radiation is simulated for a typical atmospheric window wavelength of $10.8 \mu \mathrm{m}$.

Optical properties for water droplets are generated for all wavelengths using Mie calculations (Wiscombe, 1979, revised 1996). For ice particles the parameterisation of Key et al. (2002) for a mixture of particle habits is used. The influence of gases is simulated using a standard atmosphere for the tropics from Anderson et al. (1986). For simplicity the surface is assumed to be black (albedo=0).

\subsection{Examples}

Examples for one of 52 simulation cases are presented in Fig. 4. For the whole domain of $64 \mathrm{~km} \times 64 \mathrm{~km}$ the slanted observation $\left(\theta_{\text {sensor }}=60^{\circ}\right)$ with the sun in the back of the sensor is simulated on a $250 \mathrm{~m}$ horizontal resolution. A mixture of cloud sides and tops is shown with cloud cell bottoms closer to the $\mathrm{x}$-axis than their tops. This becomes clearer for the thermal infra-red simulation at $10.8 \mu \mathrm{m}$. The warm surface emits the most infra-red radiation and is thus brightest, small cumuli and the bottoms of larger cells are cooler, the icy cloud top of the main cell (compare Fig. 2b) shows the lowest emitted radiance. The two near infra-red simulated reflectance results are similar. The reflectance is generally lower in the NIR compared to the VIS results due to absorption of water and ice particles. Most obvious difference to the $0.87 \mu \mathrm{m}$ results (and the only difference between the two NIR simulations) is the reflectance from all higher parts of 

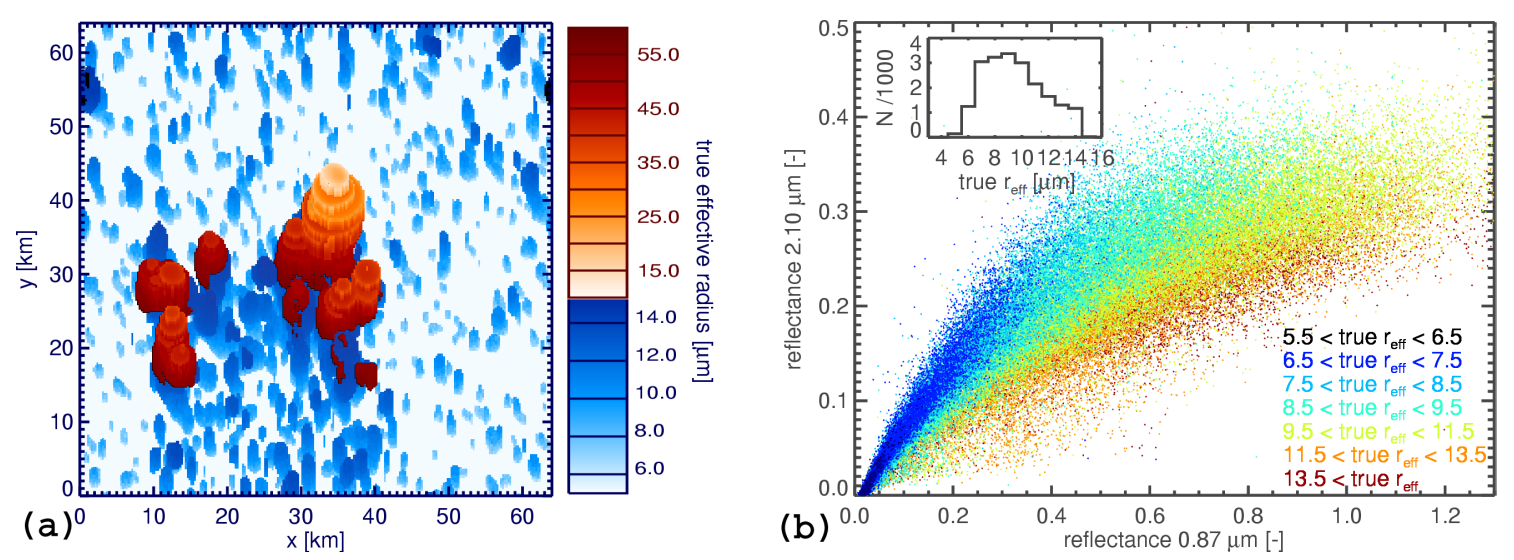

Fig. 5. (a) "true" effective radius from the GCE cloud edges for viewing zenith of $60^{\circ}$ for droplets (blue) and ice particles (red). Smallest droplet radii are at the bottom of the cloud cells, largest droplets around $15 \mu \mathrm{m}$ to the top of the liquid water volume just below the largest ice effective radius values $(\approx 60 \mu \mathrm{m})$, smaller ice effective radius is found to the top of the ice region and towards the edges of cloud volume. (b) Observed reflectance values for 0.87 and $2.1 \mu \mathrm{m}$ (Fig. 4) for certain true effective radius ranges (only droplets). The inserted histogram illustrates the underlying effective radius distribution.

large cloud cells. Especially the large ice particles are strong absorbers which leads to the even darker areas in the ice regions. These areas are the ice dominated parts as shown in Fig. 9a.

Prerequisite of the method proposed by Marshak et al. (2006) and Martins et al. (2007) is that the signature of the true effective particle size is detectable in the observable reflectance at 0.87 and $2.1 \mu \mathrm{m}$ in a statistical sense. This can now be checked for this example. Figure 5a shows the "truth", the value of effective radius which is visible for the given observational perspective in the GCE cloud structure. Figure $4 \mathrm{a}$ and $\mathrm{b}$ show the corresponding simulated reflectance.

Due to the complexity of 3-D cloud structure and 3-D radiative transfer a wide range of possible reflectance values in the VIS and NIR wavelength range occurs for each value of effective radius (Fig. 5b, only droplets). This differs clearly from the classical picture of 1-D radiative transfer through plane-parallel clouds where a clear deterministic one to one relation of a pair of VIS and NIR reflectance to one pair of optical thickness and effective radius is given for the same surface, viewing, and illumination conditions (Nakajima and King, 1990; Platnick et al., 2003). A comparison of Fig. 5b to Fig. 6 in Marshak et al. (2006) reveals that the more realistic complexity of the CRM data further increases the range of possible reflectance values, i.e. further blurs the separation of different effective radius values. Nonetheless, the core information of droplet size is still visible in the reflectance picture. There is clear evidence that smaller NIR reflectance are related to larger effective radius (for the ice region the picture is qualitatively similar).

\subsection{Database}

The next important step to check is, if this clear signature stays detectable once a range of very different convective cloud situations is mixed in one database, i.e. if there is a systematic statistical relation, or if this relation is depending on the specifics of each single cloud scene. The size of the database, on the other hand, has to be considerable to provide a statistically sound basis for a general retrieval. In the following, results for the whole data set of 52 simulated observation scenes based on as many independent realisations of cloud structures from the CRM for different stages of convective development are presented.

About 880000 simulated single pixel reflectance values for liquid water clouds and about 160000 values for ice clouds enter the database together with their respective true effective radius values from the CRM. Figures 6 and 7 give an overview of the database for liquid water and ice particles, respectively. Each plot (a) to (d) shows example distributions of true effective radius for a given combination of simulated VIS $0.87 \mu \mathrm{m}$ and NIR $2.1 \mu \mathrm{m}$ reflectance (each plot stands for one VIS reflectance and up to 5 different NIR reflectances). Not only one value of effective radius leads to a certain combination of VIS/NIR reflectance (as in 1-D radiative transfer theory), but a range of effective radius values. For each VIS/NIR bin, the occurrence of true effective radius is registered in such a frequency distribution. Panels (e) and (f) summarise all distributions in our database with more than 20 values of effective radius: The distributions' mean effective radius values for several VIS/NIR reflectance bins (different VIS reflectances in colours) is given in and Figs. 6e and $7 \mathrm{e}$ and the related distributions' standard deviations in Figs. 6f and $7 f$. 

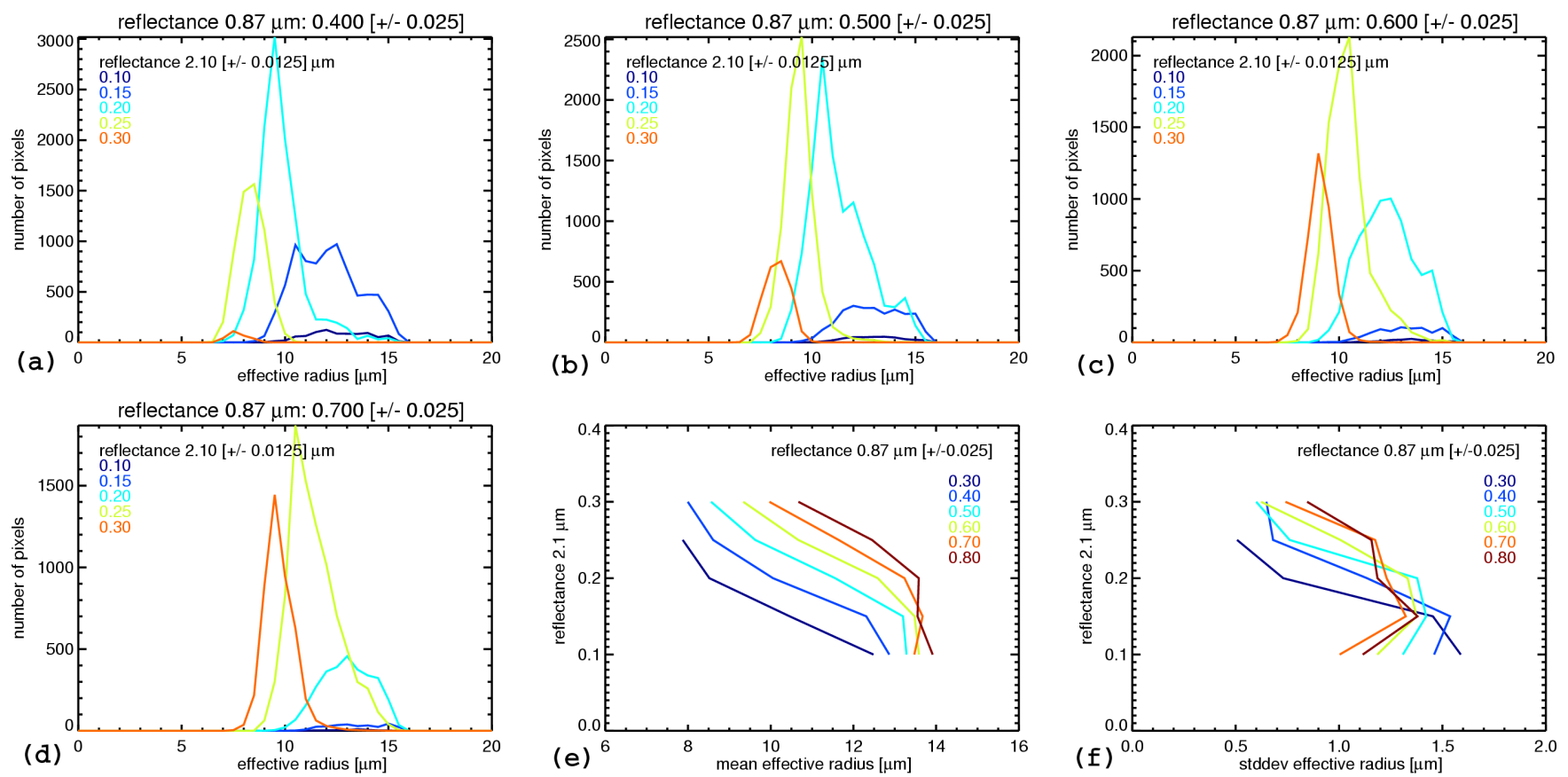

Fig. 6. (a-d) Example distributions of true effective radius for different simulated NIR/VIS reflectance bins from the simulation database for liquid water droplets. Each plot gives distributions for one VIS bin and several NIR bins (colours stand for different NIR bins). (e) Dependence of mean effective radius and (f) related standard deviation on NIR reflectance for several VIS reflectance bins (only VIS/NIR bins with at least 20 values are shown, here colours stand for different VIS bins).

In agreement with the theoretical 1-D radiative transfer picture, a clear inverse dependence of NIR reflectance on the mean value of the true effective radius distributions is visible in most cases for droplets and ice particles. For some values of reflectance the relation of VIS/NIR reflectance to effective radius is very clear, the related distributions are narrow, their standard deviations small. For other reflectance combinations the dependence is less obvious, distributions are much wider. Most distributions have a clear single peak for each VIS/NIR bin while some of the distributions for the ice particle effective radius show multiple peaks and are generally broader than the liquid droplet distributions. This causes larger relative values of standard deviation. Nonetheless, the information of cloud effective radius is clearly conserved in the observable reflectance values for ice particles as well. Also the accumulation of results from very different convective cloud cases does not blur the characteristic relations. Not shown tests confirm that the average relation of reflectance and mean effective radius is similar for different cases, and thus the distributions become increasingly stable, if more cases are added.

\section{Detection of thermodynamic phase and Bayesian re- trieval of cloud particle size}

\subsection{Phase}

From Fig. 9 the possibility of a discrimination of ice and water clouds using the NIR ratio is obvious. The absorption of ice particles drops abruptly from 2.1 to $2.25 \mu \mathrm{m}$ while the absorption by water droplets changes only slightly. Thus the reflectance of water cloud regions is only slightly smaller at 2.1 compared to $2.25 \mu \mathrm{m}$, while the reflectance of ice regions at 2.1 is much smaller than at $2.25 \mu \mathrm{m}$. The ratio is close to 0.8 for water clouds as the reflectance between 2.1 and $2.25 \mu \mathrm{m}$ changes only slightly; for ice clouds the ratio is close to 0.4 as their reflectance clearly increases at $2.25 \mu \mathrm{m}$. The output from the cloud resolving model allows for the check and optimisation of detection thresholds for a thermodynamic phase retrieval by comparison to the "truth" (Fig. 9): if the NIR ratio is larger than 0.75 , the cloud is water, if the ratio is smaller than 0.6 , an ice cloud is very likely, between these two thresholds a cloud has to be considered of uncertain (possibly mixed) phase.

\subsection{Effective radius}

The distributions presented in Fig. 6 and 7 already represent the basis for the Bayesian retrieval of cloud particle size as 

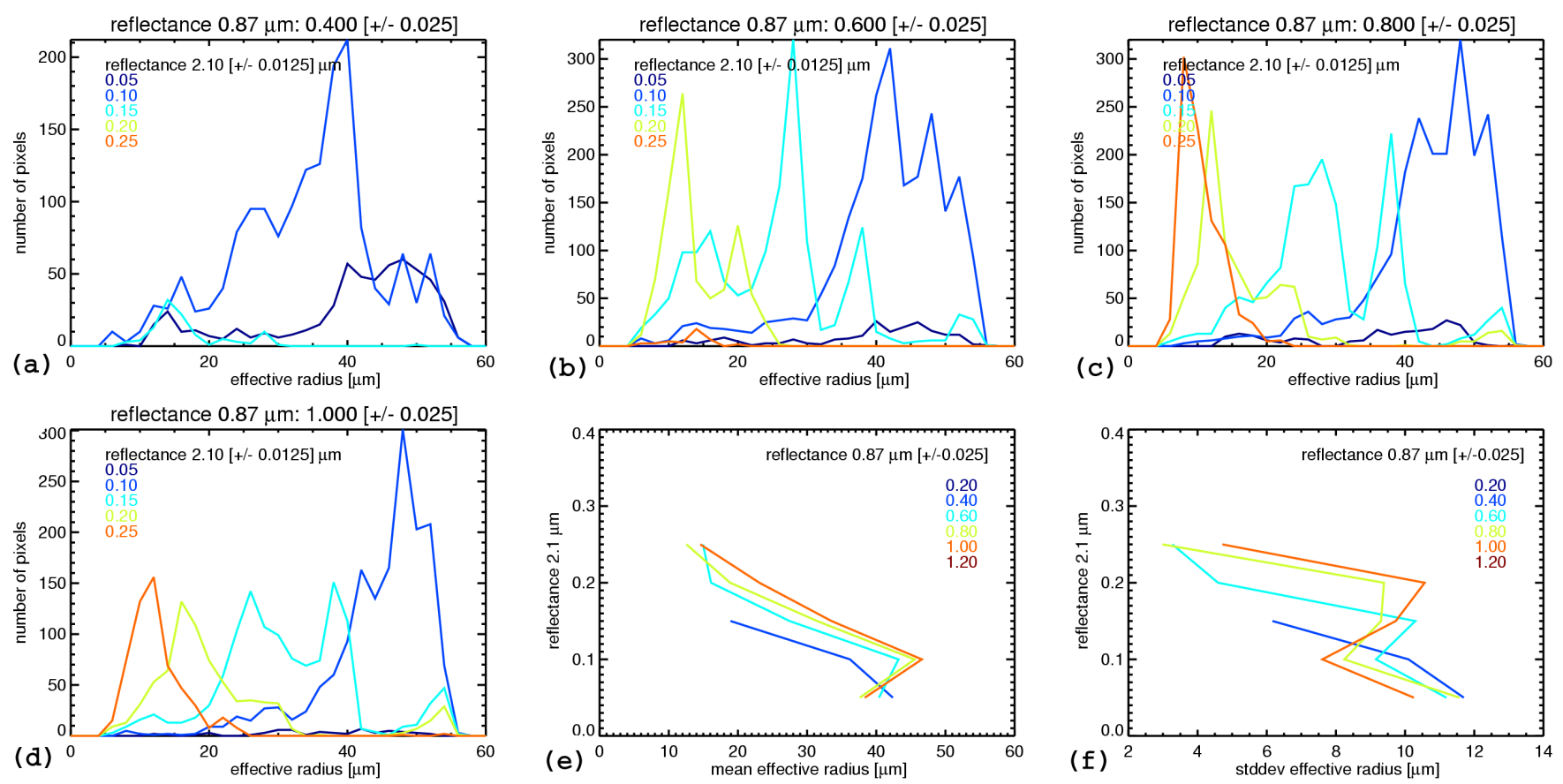

Fig. 7. Distributions and statistics for ice particles (comp. Fig. 6).

introduced in Marshak et al. (2006), as well as by McFarlane et al. (2002) and Evans et al. (2002) for other cloud property retrievals. The occurrence of certain values of effective radius in each bin of forward simulated VIS and NIR reflectance corresponds to the likelihood to have a certain effective radius for a given combination of observed VIS and NIR reflectance. This constitutes a probabilistic solution to the radiative transfer inversion problem. It can be formulated using Bayes' theorem for conditional probabilities:

$p\left(r_{\mathrm{eff}} \mid I_{\mathrm{VIS}}, I_{\mathrm{NIR}}\right)=\frac{p\left(I_{\mathrm{VIS}}, I_{\mathrm{NIR}} \mid r_{\mathrm{eff}}\right) p\left(r_{\mathrm{eff}}\right)}{\int p\left(I_{\mathrm{VIS}}, I_{\mathrm{NIR}} \mid r_{\mathrm{eff}}\right) p\left(r_{\mathrm{eff}}\right) d r_{\mathrm{eff}}} .(3)$

It states that the solution of the backward problem, the probability density $p\left(r_{\text {eff }} \mid I_{\mathrm{VIS}}, I_{\mathrm{NIR}}\right)$ of having a certain effective radius given the reflectance $I_{\mathrm{VIS}}$ and $I_{\mathrm{NIR}}$ is related to the solution of the forward problem, the probability density to observe a combination of $I_{\mathrm{VIS}}$ and $I_{\mathrm{NIR}}$ for a given cloud effective radius $p\left(I_{\mathrm{VIS}}, I_{\mathrm{NIR}} \mid r_{\mathrm{eff}}\right)$. In order to get the right probability the occurrence of $r_{\text {eff }}$ in real clouds $p\left(r_{\text {eff }}\right)$ is needed to weigh the forward result. This is closely related to the above discussed considerations about the stability of the found statistical relations over different cloud cases in terms of the width of the found distributions. The integral in the denominator on the right hand side is a normalising factor.

That means, starting from our forward simulated statistical relation between effective radius and VIS and NIR reflectance (Figs. 6 and 7), we can assign to each observational pair of VIS and NIR reflectance a probability density distribution from our database. After the initial step of identifying the thermodynamic phase, a retrieval will accordingly consist of a most likely value of effective radius (the mean) and a standard deviation describing the reliability of the result. Most droplet effective radius retrievals are therefore expected to be very clear with a mean value very close to the single maximum of the distributions and a standard deviation of 10 to $15 \%$ of this value. The ice effective radius values retrievable are more ambiguous. The reason for this difference is, on the one hand, the smaller number of realisations of ice particle/reflectance pairs that enter the database over a wider range of possible sizes (compare the vertical axes in Figs. 6 and $7 \mathrm{a}-\mathrm{d}$ ) and, on the other hand, cases where the impact of multiple cloud layers with an optically thin upper ice cloud layer generates ambiguities. In some cases the mean value thus is located between two more likely effective radius values (local maxima). Nonetheless, the standard deviation for ice effective radius retrievals is still much smaller than the retrieved mean values (20-30\% of the mean values) and thus meaningful retrievals are expected.

A possibility to include additional observed information lies in the $10.8 \mu \mathrm{m}$ data. The temperature information is not only a measure for the vertical position of the observed cloud region, but also contains information on the general cloud geometry observed. A strong local gradient of $10.8 \mu \mathrm{m}$ radiance along the direction from cloud bottom to top is evidence of a cloud side. If there is no spatial temperature gradient at all, a cloud area of constant height is in the field of view (e.g. cloud tops, Fig. 1). Of course, such geometric orientation 

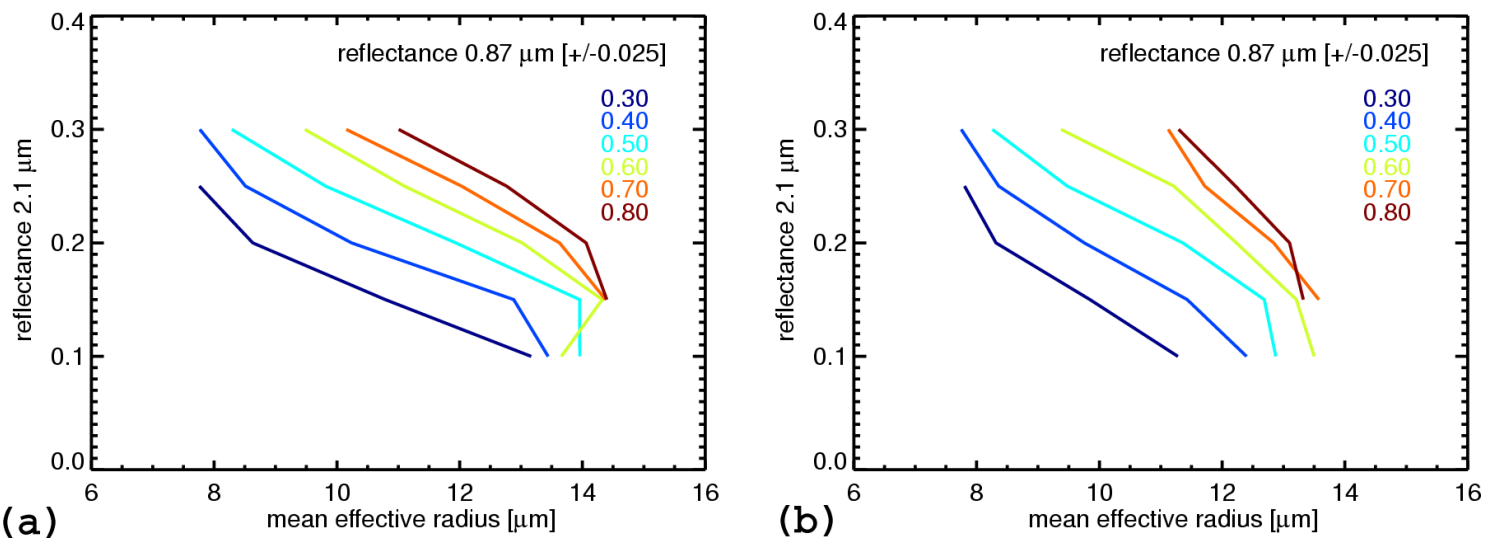

Fig. 8. Mean values of effective radius distributions for different reflectance bins (comp. Fig. 6e) for sub-classes of the whole database. (a) Relation for all simulations showing no negative local temperature gradient (cloud tops); (b) relation for all simulations showing a clear negative vertical temperature gradient (cloud sides).

has important impact on the observed reflectance as the illumination and viewing geometry is changed by up to $90^{\circ}$. The same range of effective radius values for a flat cloud top is related to a different reflectance signature compared to a steep cloud side. This fact is neglected in our database so far, but can be used to confine the application of the retrieval to suited parts of the database.

Figure 8 shows the effect of a subdivision of the database in different classes using the local vertical gradient in $10.8 \mu \mathrm{m}$ radiance, i.e. temperature. Presented are the mean values for two of five classes defined in a way to obtain equally populated classes between "no slope" (no temperature gradient, horizontal cloud tops) and "steep slope" (strong negative temperature gradient, vertical cloud sides). In the presented sub-classes slightly different values of mean effective radius are related to the same VIS/NIR reflectance bins. For example, the VIS/NIR reflectance of $0.3 / 0.1$ would lead to a retrieval of $13 \mu \mathrm{m}$ for the cloud tops while for the cloud sides the result should rather be $11 \mu \mathrm{m}$. On the other hand this division of the database into smaller samples leads to larger standard deviations (less reliability). Thus the database has to be big enough to allow a meaningful subdivision in a way that the narrowing of the distributions through selection of a suited sub-class of comparable reflectance signature is not compensated for by the increasing statistical uncertainty. This will be investigated in the context of the following test case.

\section{Test case}

In this section we demonstrate the capabilities of the retrieval using a simulated test case. For the test a different CRM microphysical model setup is used (see Sect. 2.1). This way an almost independent case is generated whose combination of microphysical setup, cloud geometry, and related observable radiation field is not part of the retrieval database (Fig. 10a). The related observations are simulated for all four wavelengths. Then the detection of thermodynamic phase and the Bayesian retrieval of effective radius is applied to the simulated observations (Fig. 10b) and is compared to the original cloud properties given in the GCE cloud data.

Figure 10c summarises the test. Depicted is information on thermodynamic phase and effective radius from the GCE cloud data set along one line at $x=32 \mathrm{~km}$. Large values of effective radius are related to ice particles, small values to water droplets. In mixed phase regions only the size of droplets is shown. Following the $x=32 \mathrm{~km}$ line through the simulated observation the effective radius profiles along the cloud sides can be seen. Increasing droplet size is visible when the sensor probes higher and higher parts of a cloud side. Once the scan along the line reaches the ice part in a vertical direction the effective radius jumps to the size of the largest ice particles at the bottom of the ice region and then decreases the higher the probed region is located in the ice cloud. In the shown case the effective size drops to very small sizes once the top edge of the cloud is left behind. A few smaller liquid water cloud cells show up behind the main convective cell $(y=55 \mathrm{~km}$ and $60 \mathrm{~km})$.

This is the kind of information retrievable from the cloud scanner sensor with a $60^{\circ}$ forward viewing zenith angle flying over the cloud scene from $y=0 \mathrm{~km}$ to $y=64 \mathrm{~km}$ (compare Fig. 10a and b). The first retrieval step is the identification of cloud phase. The result of our detection method is contrasted with the true phase (Fig. 10c). In most cases the identification of phase using the NIR ratio works accurately, even the mixed phase region around $y=31 \mathrm{~km}$ is identified adequately as "uncertain". Some parts of the ice cloud are not positively identified but classified as "uncertain" and a small part at the cloud edge even as liquid water. The reason for this misidentification could be the influence of clear areas or water clouds behind the optically thinner top edges of the ice cloud. 

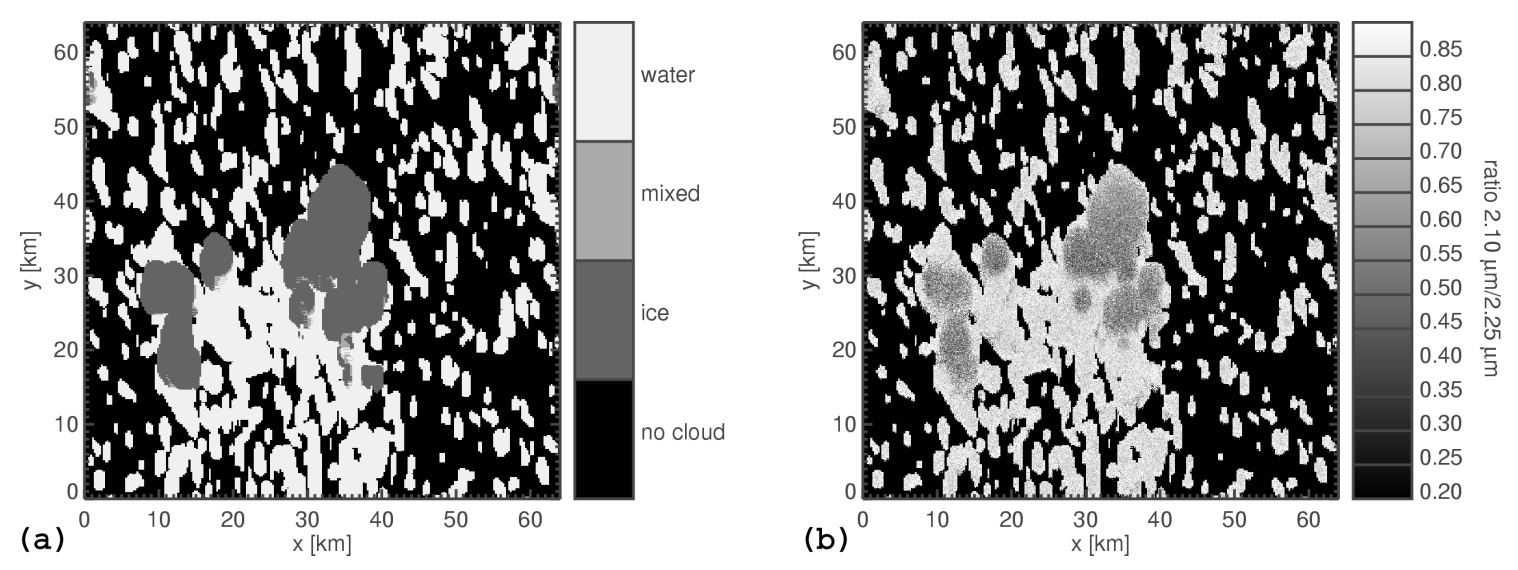

Fig. 9. Separation of thermodynamic phase. (a) cloud phase from the GCE data set for a $60^{\circ}$ viewing zenith angle, GCE volume visible from that perspective only containing liquid water is labeled "water", volume containing only ice water content is "ice", volume containing both is "mixed". (b) Ratio of reflectance 2.1/2.25 $\mu \mathrm{m}$. Due to differences in ice absorption, the cloud phase is separable.

Table 1. Retrieval errors for droplet and ice particle size (root mean square, bias, and standard deviation) in $\mu \mathrm{m}$ for the standard retrieval and the retrieval using the spatial gradient at $10.8 \mu \mathrm{m}(*)$.

\begin{tabular}{lcccccc}
\hline & rms & rms* & bias & bias* & $\sigma$ & $\sigma *$ \\
\hline water & 1.639 & 1.608 & 0.091 & 0.136 & 1.042 & 1.002 \\
ice & 8.062 & 8.319 & -0.697 & -0.797 & 8.665 & 7.826 \\
\hline
\end{tabular}

After identification of the cloud phase the Bayesian effective radius retrieval is applied separately for each phase. "Uncertain" regions are omitted. Both versions of the retrieval, the one using the complete database and the one using the spatial gradient in the $10.8 \mu \mathrm{m}$ observation as additional information, are able to reproduce the gradient of effective radius along the vertical profile on this very high spatial resolution of $250 \mathrm{~m}$. The result is very good for some of the shallow cumuli (e.g. at $y=10 \mathrm{~km}$ or $60 \mathrm{~km}$ ). Already on this high spatial resolution the true gradient is closely reproduced. In other regions the averaging over a few nearby observations would be enough to reach this quality. The retrieval is less clear for the ice cloud areas where the phase was identified correctly in the first place. The average effective radius is measured accurately and even some of the profile features seem to be detected, but there are larger pixel to pixel fluctuations in the retrieval, probably caused by the ambiguities in some of the size distributions in the database (Fig. 7). That means, averaging over slightly larger areas had to be done to reach a clear result for the ice particle size profile.

The second output of the Bayesian retrieval is the standard deviation describing the accuracy of each retrieval. These values are always very close to $\sigma=1 \mu \mathrm{m}$ for the droplet retrievals. As expected the standard deviation for ice particles is larger, between 7 and $9 \mu \mathrm{m}$. Small improvements of the retrieval are visible if the information on the spatial gradient at $10.8 \mu \mathrm{m}$ is used as additional information to select the adequate part of the complete retrieval database especially for ice clouds. The pixel to pixel fluctuation is smaller and the retrieved standard deviation is decreasing accordingly.

Table 1 provides average error values of retrievals for the full scene in Fig. 10a and b. Root mean square error, overall bias, and the average retrieved standard deviation are given for 26000 retrievals of droplet effective radius and 360 retrievals of ice particle effective radius, whenever the cloud phase was detected correctly. The values are similar to the ones in Fig. 10c. A retrieval for a single pixel has a typical root mean square error of about $1.6 \mu \mathrm{m}$ for water and $8 \mu \mathrm{m}$ for ice particles which had to be expected from the retrieved values of standard deviation. The retrieval version using the thermal gradient as additional geometry measure does not show a clear improvement over the whole scene.

\section{Conclusions}

We presented a thorough check of the proposed cloud scanner remote sensing approach for the retrieval of profiles of thermodynamic phase and effective radius (Martins et al., 2007) and provided a first experimental retrieval database based on a large number of cloud cases. It was demonstrated that it is possible to measure highly accurate high spatial resolution cloud properties from the proposed cloud scanner perspective.

For this purpose, 52 cloud data sets from cloud resolving model and a 3-D radiative transfer model were used to provide an extensive set of simulated cloud scanner observations. It was shown that regardless of the high spatial complexity of realistic cloud structures and 3-D radiative 

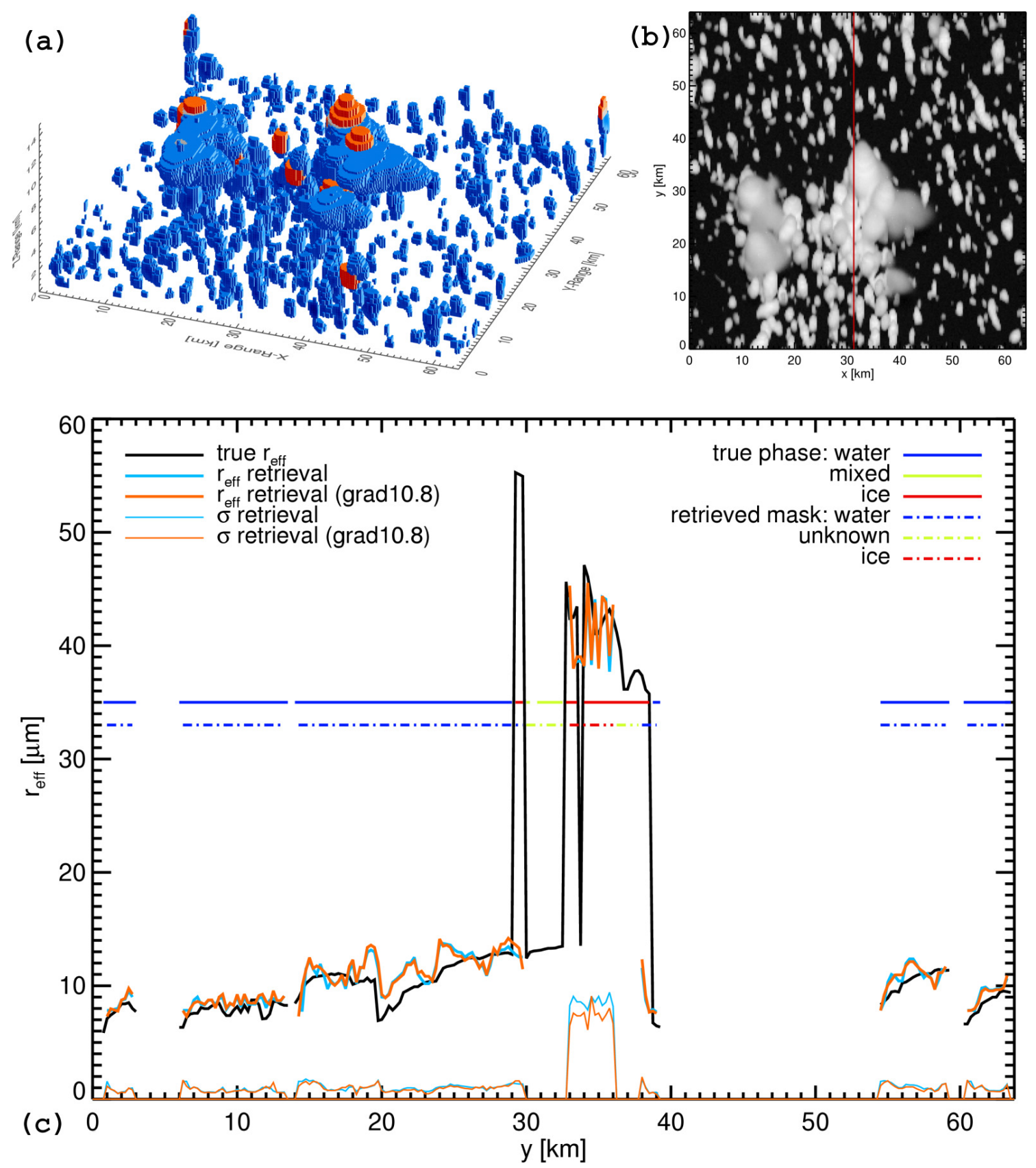

Fig. 10. (a) 3-D distribution of LWC (blue) and IWC (red) for the test cloud data set. (b) Related simulated observation of $0.87 \mu \mathrm{m}$ reflectance for $60^{\circ}$ viewing zenith and $45^{\circ}$ solar zenith angle (to the bottom of the image). (c) Result of two retrieval versions applied to one line of measurements from the simulated data - the red line in (b) - at four wavelengths: The true effective radius along the sides and tops of the GCE cloud data set at $x=32 \mathrm{~km}$ for the given viewing geometry is shown as thick black line. Values above $15 \mu \mathrm{m}$ are of ice particles (indicated by the thick blue line), effective radius below $15 \mu \mathrm{m}$ mostly belongs to cloud edges with only water droplets (thick red line). The results of the phase retrievals are shown with thick broken lines, the results of the effective radius retrievals are shown in orange and light blue (mean effective radius in thick lines, related standard deviation in thin lines at the bottom of the figure).

transfer, the signatures of cloud particle effective radius and cloud phase were clearly detectable in a statistical sense. This fact is used to employ the simulation database as starting point of a Bayesian retrieval of effective radius accounting for realistic cloud structure and 3-D radiative transfer (Marshak et al., 2006). 

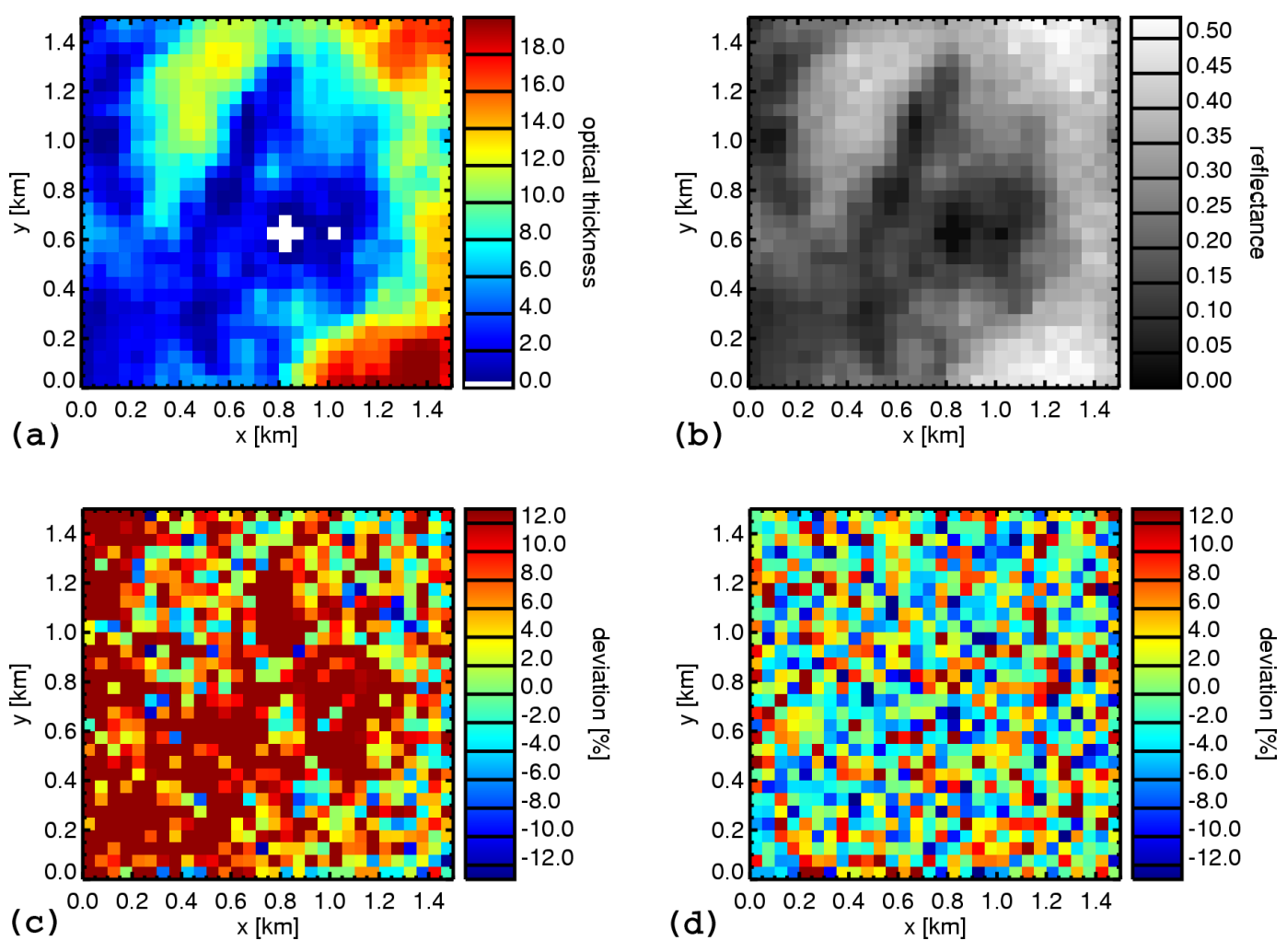

Fig. A1. Comparison of MYSTIC simulations for an example cloud scene (solar zenith of $5^{\circ}$, nadir view): (a) field of optical thickness and (b) related reference simulation, (c) deviation from this reference field for simulations with delta-scaling of the phase function for all scattering events and (b) for delta-scaling starting with the third scattering (scene biases are $8 \%$ and $0.6 \%$, respectively). Note the dependency of the deviation on the optical thickness in (c) and its absence in (d).

Given the assumption that the relevant physical connection of particle size and observed radiance is represented in the simulated database for all possible cloud situations, only statistical uncertainties in single retrievals would be expected, but no bias. An independent test checking the influence of variations in cloud structure and microphysics was used to test the capabilities of the approach. The results of this test are very promising. Only a minimal overall bias was found for the example cloud scene in the order of $1 \%$ for droplets and ice particles. For measurements on the high $250 \mathrm{~m}$ resolution, the retrieved uncertainty from our database and the actual statistical error for our test case was found to be in the order of $1 \mu \mathrm{m}$ for droplets and $8 \mu \mathrm{m}$ for ice particles. This is an acceptable uncertainty as the usual values of effective radius are several times larger. It is a clear improvement over classical plane-parallel deterministic retrievals which would have a much higher pixel to pixel uncertainty and bias for the same scene (tested but not shown in detail for droplets in $1 \mathrm{~km}$ cloud covered pixels: $\mathrm{rms}=8 \mu \mathrm{m}$, bias $=2 \mu \mathrm{m}$ ). Such values would completely prohibit any attempt to derive a correct high resolution microphysics profile.

The used cloud cases were not entirely independent, but related to each other regarding the overall cloud type and mi- crophysical structure. In addition, some of the microphysical assumptions restrained the possible variability in our cloud cases compared to stronger thinkable variations in droplet size in nature, e.g. in areas far from updraft regions strongly affected by mixing. Nonetheless, the useful application of a retrieval database generated under such slightly simplified conditions is thus not necessarily limited to likewise cloud cases. On the one hand a careful pre-selection of cloud type where the database is applicable is possible. On the other hand the retrieval is still likely to work for an even wider range of real cases with respect to the retrieved effective radius values, whereas the retrieved pixel uncertainty values might then underestimate reality.

A proof-of-concept for the statistical Bayesian retrieval and the cloud side scanner approach for the derivation of cloud particle size profiles in a natural complex observation situation is provided. A possibility to improve the retrieval by selecting the suited part of the information mixture in the retrieval database regarding geometrical conditions was outlined. The latter technique can probably be further optimised by adjusting the TIR gradient classification. Following the Bayes' theorem a probability of occurrence of certain cloud structures in nature should become part of the retrieval in the 
future ( $p\left(r_{\text {eff }}\right)$ in Eq. 3). This information could be taken from convective cloud climatologies to weigh the cases in the database.

Next the retrieval database has to be extended to more solar illumination geometries - due to the need for 3-D Monte Carlo simulations this is a computationally still very demanding effort. Real measurements from aircraft as the ones taken by Martins et al. (2007) have already been collected and will be evaluated once a sufficiently complete database is obtained. Once this is done on more than a case study base, new insights into convective cloud physics and its interaction with aerosol will be gained.

\section{Appendix A}

\section{Variance reduction techniques for the Monte Carlo model}

\section{A1 Thermal backward}

Backward simulations are always used, when a forward simulation would "waste" the majority of the simulation time to trace photons which do not contribute to the result (see, e.g. Marshak and Davis, 2005). For a thermal radiative transfer calculation, the majority of photons emitted according to the Planck function everywhere in the atmosphere never reach the sensor since absorption is high especially in clouds. Thus, we extended MYSTIC based on the existing backward solution (Emde and Mayer, 2007) for the thermal simulations needed in this paper. Photons are started from the sensor in the direction for which the radiance is calculated, photons are tracked until they are absorbed, and the value of the Planck function at this point is collected as contribution to the result. Comparisons of MYSTIC and DISORT (Stamnes et al., 1988) show differences below $0.1 \%$ for a plane-parallel cloud test cases. Computation time for 3-D simulations of radiance to reach an accuracy of about $0.1 \%$ is only double the time needed for plane-parallel 1-D DISORT calculations and thus well within feasible time limits.

\section{A2 Solar delta-scaling}

Strongly forward peaked scattering functions for cloud droplets in the solar wavelength range cause larger uncertainties in Monte Carlo calculations of radiative transfer. Antyufeev (1996) introduces delta-scaling of the phase function peak and a related re-scaling of the scattering coefficients as a possible method to minimise these uncertainties in Monte Carlo models. We approximated the Mie phase function $P(\mu)$ with $P_{\mathrm{ds}}(\mu)$ by replacing the forward peak for scatter angles $\mu=\cos (\theta) \geq 1-\epsilon$ (small scatter angles) by a delta-function, i.e.,

$$
P(\mu) \approx P_{\mathrm{ds}}(\mu)=q \delta(1-\mu)+(1-q) P_{\epsilon}^{*}(\mu)
$$

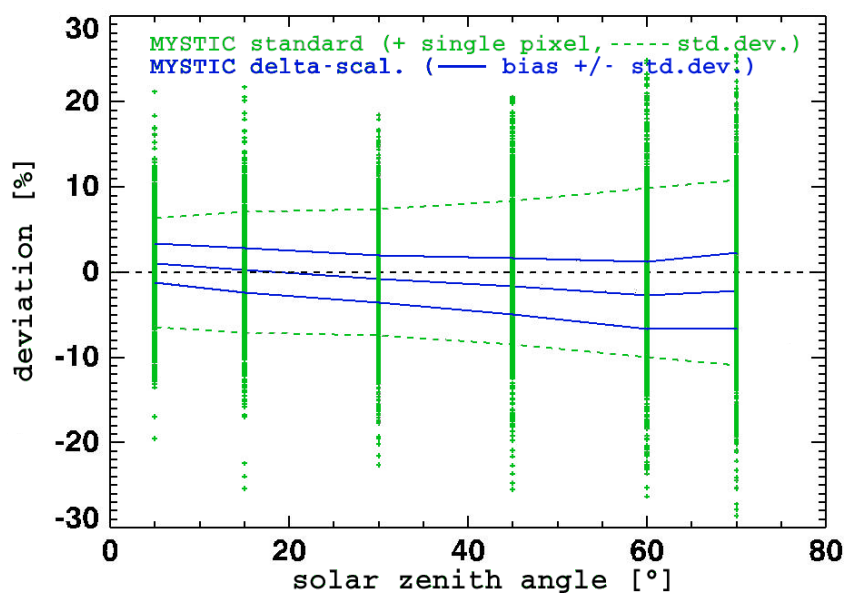

Fig. A2. Comparison of scatter (standard deviation) and bias (compared to a reference simulation) for simulations without deltascaling (green) and with delta-scaling starting with the third scatter event (blue) - the time effort for both simulations is the same. Shown are single pixel errors (+) and the related standard deviation (broken lines) for the run without delta-scaling, as well as the standard deviation and bias for the run with delta-scaling (black lines). The bias of using delta-scaling is clearly within the range of uncertainty that has to be expected for simulations without delta-scaling for the same time effort. The time for a standard simulation to reach the same level of uncertainty as the simulation with delta-scaling would be 5-10 times longer than the one presented.

with $\epsilon$ as an adjustable parameter and where

$P_{\epsilon}^{*}(\mu)=\left\{\begin{array}{cc}0 & , 1 \geq \mu \geq 1-\epsilon \\ \frac{1}{1-q} P(\mu), & 1-\epsilon>\mu \geq-1\end{array}\right.$

and $q=\int_{1-\epsilon}^{1} P(\mu) d \mu$. The new extinction coefficient is $\beta^{*}=\beta\left(1-q \omega_{0}\right)$ where $\beta$ is the original extinction coefficient and $\omega_{0}$ the single scattering albedo. A given level of accuracy is thus reached in a substantially shorter period of time especially for optically thick cases. Iwabuchi (2006) demonstrates this variance reduction method and the theoretical background, but also emphasises that the approach causes small biases, which depend on zenith angle and optical thickness (Fig. A1). Deviations are always caused due to effects of the first orders of scattering, since in a 3-D cloud scene the first scattering events define a considerable redirection of photon incidence. Thus we implemented the delta-scaling into MYSTIC in a way that first and second order scattering are treated with un-scaled phase function and scattering coefficient and scaling is only applied to all higher orders of scattering (Fig. A1). By accepting a small correctable bias only depending on the illumination geometry, the simulation time frame to reach the required level of accuracy $(3 \%)$ is reduced by a factor 5 to 10 depending on the average scene optical thickness (Fig. A2).

Acknowledgements. The authors want to thank W.-K. Tao and $\mathrm{X}$. Li for their support and helpful discussions and M. Pfeifer 
for her critical review of the manuscript. T. Zinner was funded by a German Research Foundation (DFG) Fellowship and the NASA visiting scientists program. This work was also supported by the United States Department of Energy (under grant DEA105-90ER61069 to NASA GSFC) as part of the Atmospheric Radiation Measurements program (ARM). The contribution of three anonymous referees to the final form of the manuscript is gratefully acknowledged.

Edited by: Y. Balkanski

\section{References}

Albrecht, B. A.: Aerosols, cloud microphysics and fractional cloudiness, Science, 245, 1227-1230, 1989.

Anderson, G., Clough, S., Kneizys, F., Chetwynd, J., and Shettle, E.: AFGL atmospheric constituent profiles, Tech. Rep. AFGLTR-86-0110, AirForce Geophys. Lab., 1986.

Andreae, M. O., Rosenfeld, D., Artaxo, P., Costa, A. A., Frank, G. P., Longo, K. M., and Silva-Dias, M. A. F.: Smoking rain clouds over the Amazon, Science, 303, 1337-1342, 2004.

Antyufeev, V. S.: Solution of the generalized transport equation with a peak-shaped indicatrix by the Monte Carlo method, Russ. J. Numer. Anal. Math. Model., 11, 113-137, 1996.

Baker, M. B., Corbin, R. G., and Latham, J.: The influence of entrainment on the evolution of cloud droplet spectra: I. A model of inhomogeneous mixing, Q. J. Roy. Meteorol. Soc., 106, 581598, 1980.

Barker, H. W., Goldstein, R. K., and Stevens, D. E.: Monte Carlo simulation of solar reflectances for cloudy atmospheres, J. Atmos. Sci., 60, 1881-1894, 2003.

Blyth, A. M. and Latham, J.: A climatological parameterization for cumulus cloud, J. Atmos. Sci., 48, 2367-2372, 1991.

Brenguier, J.-L., Pawlowska, H., Schüller, L., Preusker, R., Fischer, J., and Fouquart, Y.: Radiative properties of boundary layer clouds: Droplet effective radius versus number concentration, J. Atmos. Sci., 57, 803-821, 2000.

Cahalan, R. F., Oreopoulos, L., Marshak, A., Evans, K. F., Davis, A. B., Pincus, R., Yetzer, K. H., Mayer, B., Davies, R., Ackerman, T. P., et al.: The I3RC: Bringing together the most advanced radiative transfer tools for cloudy atmospheres, B. Am. Meteorol. Soc., 86, 1275-1293, 2005.

Emde, C. and Mayer, B.: Simulation of solar radiation during a total eclipse: a challenge for radiative transfer, Atmos. Chem. Phys., 7, 2259-2270, 2007,

http://www.atmos-chem-phys.net/7/2259/2007/.

Evans, K. F., Walter, S. J., Heymsfield, A. J., and McFarquhar, G. M.: The Submillimeter-wave cloud ice radiometer: Simulations of retrieval algorithm performance, J. Geophys. Res., 107, 4028, doi:10.1029/2001JD000709, 2002.

Fletcher, N. H.: The Physics of Rain Clouds, Cambridge University Press, Thun, Frankfurt am Main, 1962.

French, J., Vali, G., and Kelly, R. D.: Observations of microphysics pertaining to the development of drizzle in warm, shallow cumulus clouds, Q. J. Roy. Meteorol. Soc., 126, 415-443, 2000.

Freud, E., Rosenfeld, D., Andreae, M. O., Costa, A. A., and Artaxo, P.: Robust relations between $\mathrm{CCN}$ and the vertical evolution of cloud drop size distribution in deep convective clouds, Atmos.
Chem. Phys., 8, 1661-1675, 2008,

http://www.atmos-chem-phys.net/8/1661/2008/.

Gerber, H.: Entrainment, mixing, and microphysics in RICO cumulus, in: Proceedings of the 12th Conference on Atmospheric Radiation/12th Conference on Cloud Physics, Madison, WI, July 2006.

Hallett, J. and Mossop, S. C.: Production of secondary ice particles during the riming process, Nature, 249, 26-28, 1974.

Hong, S.-Y., Dudhia, J., and Chen, S.-H.: A revised approach to ice microphysical processes for the bulk parameterization of clouds and precipitation, Mon. Weather Rev., 132, 103-120, 2004.

IPCC 2007: Climate Change 2007: The physical science basis, Intergovernmental Panel on Climate Change (IPCC) Secretariat, c/o World Meteorological Organization, Geneva, Switzerland, 2007.

Iwabuchi, H.: Efficient Monte Carlo methods for radiative transfer modeling, J. Atmos. Sci., 63, 2324-2339, 2006.

Kaufman, Y. J. and Koren, I.: Smoke and Pollution Aerosol Effect on Cloud Cover, Science, 313, 655-658, doi:10.1126/science.1126232, 2006.

Key, J., Yang, P., Baum, B., and Nasiri, S.: Parameterization of shortwave ice cloud optical properties for various particle habits, J. Geophys. Res., 107, 4181, doi:10.1029/2001JD000742, 2002.

Khain, A. P., Rosenfeld, D., and Pokrovsky, A.: Simulating convective clouds with sustained supercooled liquid water down to $-37.5 \mathrm{C}$ using a spectral microphysics model, Geophys. Res. Lett., 28, 3887-3890, 2001.

Lang., S., Tao, W.-K., Cifelli, R., Olson, W., Halverson, J., Rutledge, S., and Simpson, J.: Improving simulations of convective systems from TRMM LBA: Easterly and westerly regimes, J. Atmos. Sci., 64, 1141-1164, 2007.

Marshak, A. and Davis, A. B. (Eds.): 3D Radiative Transfer in Cloudy Atmospheres, Springer, Berlin, 1 edn., 2005.

Marshak, A., Martins, J. V., Zubko, V., and Kaufman, Y. J.: What does reflection from cloud sides tell us about vertical distribution of cloud droplet sizes?, Atmos. Chem. Phys., 6, 5295-5305, 2006, http://www.atmos-chem-phys.net/6/5295/2006/.

Martin, G. M., Johnson, D. W., and Spice, A.: The measurement and parameterization of effective radius of droplets in warm stratocumulus clouds, J. Atmos. Sci., 51, 1823-1842, 1994.

Martins, J. V., Marshak, A., Remer, L. A., Rosenfeld, D., Kaufman, Y. J., Fernandez-Borda, R., Koren, I., Zubko, V., and Artaxo, P.: Remote sensing the vertical profile of cloud droplet effective radius, thermodynamic phase, and temperature, Atmos. Chem. Phys. Discuss., 7, 4481-4519, 2007, http://www.atmos-chem-phys-discuss.net/7/4481/2007/.

Mayer, B.: I3RC phase 1 results from the MYSTIC Monte Carlo model, in: Intercomparison of three-dimensional radiation codes: Abstracts of the first and second international workshops, University of Arizona Press, ISBN 0-9709609-0-5, 1999.

Mayer, B.: I3RC phase 2 results from the MYSTIC Monte Carlo model, in: Intercomparison of three-dimensional radiation codes: Abstracts of the first and second international workshops, University of Arizona Press, ISBN 0-9709609-0-5, 2000.

Mayer, B. and Kylling, A.: Technical Note: The libRadtran software package for radiative transfer calculations: Description and examples of use, Atmos. Chem. Phys., 5, 1855-1877, 2005, http://www.atmos-chem-phys.net/5/1855/2005/. 
McFarlane, S. A., Evans, K. F., and Ackerman, A. S.: A Bayesian algorithm for the retrieval of liquid water properties from microwave radiometer and millimiter radar data, J. Geophys. Res., 107, 4317, doi:10.1029/2001JD001011, 2002.

McFarquhar, G. M., Iacobellis, S., and Somerville, R. C. J.: SCM simulations of tropical ice clouds using observationally based parameterizations of microphysics, J. Climate, 16, 1643-1664, 2003.

Meyers, M. P., DeMott, P. J., and Cotton, W. R.: New primary icenucleation parameterization in an explicit cloud model, J. Appl. Meteorol., 31, 708-721, 1992.

Nakajima, T. and King, M. D.: Determination of the optical thickness and effective particle radius of clouds from reflected solar radiation measurements. Part I: Theory, J. Atmos. Sci., 47, 18781893, 1990.

Platnick, S., King, M. D., Ackerman, S. A., Menzel, W. P., Baum, B. A., Riedi, J. C., and Frey, R.: The MODIS cloud products: Algorithms and examples from TERRA, IEEE T. Geosci. Rem., 41, 459-473, 2003.

Rosenfeld, D.: ATMOSPHERE: Aerosols, Clouds, and Climate, Science, 312, 1323-1324, doi:10.1126/science.1128972, 2006.

Rosenfeld, D. and Woodley, W. L.: Satellite-based insights into the precipitation formation processes in continental and maritime convective clouds, B. Am. Meteorol. Soc., 79, 2457-2476, 1998.

Rosenfeld, D. and Woodley, W. L.: Deep convective clouds with sustained supercooled liquid water down to $-37.5^{\circ} \mathrm{C}$, Nature, 405, 440-442, 2000.

Rutledge, S. A. and Hobbs, P. V.: The mesoscale and microscale structure and organization of clouds and precipitation in midlatitude cyclones. Part XII: A diagnostic modeling study of precipitation development in narrow cold-frontal rainbands, J. Atmos. Sci., 41, 2949-2972, 1984.

Stamnes, K., Tsay, S. C., Wiscombe, W., and Jayaweera, K.: A numerically stable algorithm for discrete-ordinate-method radiative transfer in multiple scattering and emitting layered media, Appl. Optics, 27, 2502-2509, 1988.
Stith, J. L., Dye, J. E., Bansemer, A., Heymsfield, A. J., Grainger, C. A., Petersen, W. A., and Cifelli, R.: Microphysical observations of tropical clouds, J. Appl. Meteorol., 41, 97-117, 2002.

Tao, W.-K. and Simpson, J.: The Goddard Cumulus Ensemble Model. Part I: Model description, Terr. Atmos. Oceanic Sci., p. 1954, 1993.

Tao, W.-K., Simpson, J., Baker, D., Braun, S., Chou, M.-D., Ferrier, B., Johnson, D., Khain, A., Lang, S., Lynn, B., et al.: Microphysics, radiation and surface processes in the Goddard Cumulus Ensemble (GCE) model, Meteorol. Atmos. Phys., 82, 97-137, 2003.

Twomey, S.: The influence of pollution on the shortwave albedo of clouds, J. Atmos. Sci., 34, 1149-1152, 1977.

Williams, E., Rosenfeld, D., Madden, M., Gerlach, J., Gears, N., Atkinson, L., Dunnemann, N., Frostrom, G., Antonio, M., Biazon, B., et al.: Contrasting convective regimes over the Amazon: Implications for cloud electrification, J. Geophys. Res., 107, 8082, doi:10.1029/2001JD000380, 2002.

Wiscombe, W. J.: Mie scattering calculations: advances in technique and fast, vector-speed computer codes, Tech. rep., National Center for Atmospheric Research, Boulder, CO, USA, 1979, revised 1996.

Wyser, K.: The effective radius in ice clouds, J. Climate, 11, 1793 1802, 1998.

Zinner, T. and Mayer, B.: Remote sensing of stratocumulus clouds: Uncertainties and biases due to inhomogeneity, J. Geophys. Res., 111, D14 209, doi:10.1029/2005JD006955, 2006.

Zinner, T., Mayer, B., and Schröder, M.: Determination of threedimensional cloud structures from high-resolution radiance data, J. Geophys. Res., 111, D08204, doi:10.1029/2005JD006062, 2006. 